\title{
Optimal stopping problems for running minima with positive discounting rates
}

\author{
Pavel V. Gapeev*
}

\begin{abstract}
We present analytic solutions to some optimal stopping problems for the running minimum of a geometric Brownian motion with exponential positive discounting rates. The proof is based on the reduction of the original problems to the associated free-boundary problems and the solution of the latter problems by means of the smooth-fit and normal-reflection conditions. We show that the optimal stopping boundaries are determined as the minimal solutions of certain first-order nonlinear ordinary differential equations. The obtained results are related to the valuation of perpetual dual American lookback options with fixed and floating strikes in the Black-Merton-Scholes model from the point of view of short sellers.
\end{abstract}

\section{Introduction}

The main aim of this paper is to present analytic solutions to the discounted optimal stopping problems of (2.4) and (5.1) for the running minimum $Y$ associated with the process $X$ defined in (2.1)-(2.2) with exponential positive discounting rates. These problems are related to the option pricing theory in mathematical finance, where $X$ can describe the price of the underlying risky asset on a financial market (see, e.g. Shiryaev [39; Chapter VIII; Section 2a], Peskir and Shiryaev [33; Chapter VII; Section 25], or Detemple [9], for an extensive overview of other related results in the area). In this view, the values in (2.4) and (5.1) can be interpreted as rational (or no-arbitrage) prices of the perpetual dual American lookback options with fixed and floating strikes $L>0$ and $L X>0$ in the Black-Merton-Scholes model from the point of view of short sellers. The original perpetual Russian and American lookback options optimal stopping problems with exponential negative discounting rates were formulated and solved in Shepp and Shiryaev [36]-[37] and Pedersen [28], Guo and Shepp [22], and Beibel and Lerche [5], for a model with underlying geometric Brownian motions, as well as in [11] for a model with exponential jump-diffusion processes.

In the case of $L=0$, the problems in (2.4) and (5.1) become the dual Russian option problems for selling short, which were formulated and explicitly solved by Shepp and Shiryaev [38], by means of reducing the initial problem to an optimal stopping problem for a two-dimensional (continuous) Markov process and solving the latter problem by using the smooth-fit and normal-reflection conditions. More recently, the problems in (2.4) and (5.1) in the case of $L=0$ were solved in [13] by means of reducing the initial problems to optimal stopping problems for a one-dimensional diffusion process with reflection following the change-of-measure arguments from Shepp and Shiryaev [37]. Gerber et al. [19] and Mordecki and Moreira [26] obtained closed form solutions to the perpetual Russian option problems for diffusions with negative exponential jumps. Asmussen et al. [1] derived explicit expressions for the values of the first passage time problems associated with the prices of perpetual Russian options for Lévy processes with phase-type jumps by means of the martingale stopping and Wiener-Hopf factorisation. Avram et al. [2] studied exit problems for spectrally negative Lévy processes and applied the results to solving optimal stopping problems associated with perpetual Russian and American

${ }^{*}$ London School of Economics, Department of Mathematics, Houghton Street, London WC2A 2AE, United Kingdom; e-mail: p.v.gapeev@lse.ac.uk

Mathematics Subject Classification 2010: Primary 60G40, 60G44, 60J65. Secondary 91G20, 91B25, 35R35.

Key words and phrases: Optimal stopping problem, exponential positive discounting rate, Brownian motion, running minimum process, free-boundary problem, smooth fit, normal reflection, a change-of-variable formula with local time on surfaces, perpetual dual American lookback options.

Date: July 30, 2020 
put options. Guo and Zervos [23] and Rodosthenous and Zervos [34] derived solutions for discounted optimal stopping problems related to the pricing of perpetual American options with more general payoff functions depending on the current values of the associated running maximum processes in diffusion models for the underlying risky asset prices. Other more complicated optimal stopping problems and games within the framework of models based on spectrally negative Lévy processes and their running maxima processes were studied by Baurdoux and Kyprianou [6], Ott [27], Kyprianou and Ott [25], and Baurdoux and van Schaik [7] among others.

In the present paper, we study the optimal stopping problems of (2.4) and (5.1) for the running minimum $Y$ of the geometric Brownian motion $X$ with linear payoffs. In contrast to the dual Russian option problem studied in [37], the problem of (2.4) is necessarily two-dimensional in the sense that it cannot be reduced to an optimal stopping problem for a one-dimensional (time-homogeneous strong) Markov process. It is shown that the optimal stopping boundaries for the process $X$ can be expressed as functions of the current value of the running minimum process $Y$. We obtain explicit expressions for the value functions in (2.4) and (5.1) and apply the normal-reflection condition at the edge of the two-dimensional state space for $(X, Y)$ to characterise the optimal stopping boundaries as the minimal solutions to the appropriate first-order nonlinear ordinary differential equations. Other optimal stopping problems with exponential positive discounting rates were recently considered by Xia and Zhou [40], Battauz et al. [3]-[4], De Donno et al. [8], and [14] among others. Optimal stopping problems for three-dimensional continuous Markov processes having the running maximum or minimum as well as the running maximum drawdown or drawup as components were recently studied by Peskir [31]-[32], Glover et al. [20], and [16]-[18] among others.

The paper is organised as follows. In Section 2, we formulate the optimal stopping problem of (2.4) for the two-dimensional Markov process $(X, Y)$ related to the perpetual dual American fixed-strike lookback option problem. The latter problem is then reduced to the associated free-boundary problem for the value function which satisfies the smooth-fit condition at the optimal stopping boundary and the normal-reflection conditions at the edge of the state space of the two-dimensional process. In Section 3, we obtain analytic solutions of the free-boundary problem and derive first-order nonlinear ordinary differential equations for the candidate optimal stopping boundary under various relations between the parameters of the model. In Section 4, we apply the change-of-variable formula with local time on surfaces from Peskir [30] to verify that the resulting solution of the free-boundary problem provides the expressions for the value function and optimal stopping boundary in the initial problem. In Section 5, we present explicit solutions for the associated dual American floating-strike lookback option optimal stopping problem under various relations between the parameters of the model. The main results of the paper are stated in Theorems 4.1 and 5.1.

\section{The case of dual lookback options with fixed strikes}

In this section, we introduce the setting and notation of the two-dimensional optimal stopping problem which is related to the pricing of perpetual dual American fixed-strike lookback option and formulate an associated free-boundary problem.

2.1 Formulation of the problem. For a precise formulation of the problem, let us consider a probability space $(\Omega, \mathcal{F}, P)$ with a standard Brownian motion $B=\left(B_{t}\right)_{t \geq 0}$ and its natural filtration $\left(\mathcal{F}_{t}\right)_{t \geq 0}$. Let us define the process $X=\left(X_{t}\right)_{t \geq 0}$ by:

$$
X_{t}=x \exp \left(\left(\mu-\sigma^{2} / 2\right) t+\sigma B_{t}\right)
$$

which solves the stochastic differential equation:

$$
d X_{t}=\mu X_{t} d t+\sigma X_{t} d B_{t} \quad\left(X_{0}=x\right)
$$

where $x>0$ is fixed, and $\mu \in \mathbb{R}, r>0$, and $\sigma>0$ are some given constants. It is assumed that the process $X$ describes the price of a risky asset on a financial market, where $\mu$ is the local drift rate and $\sigma$ is the volatility rate. (Note that, in the case $\mu=-r$, which corresponds to the exponential negative inflation rates, the process $\left(e^{r t} X_{t}\right)_{t \geq 0}$ is a martingale with respect to the initial probability measure $P$.) Let us now define 
the associated with $X$ running minimum process $Y=\left(Y_{t}\right)_{t \geq 0}$ by:

$$
Y_{t}=y \wedge \min _{0 \leq s \leq t} X_{s}
$$

for an arbitrary $0<y \leq x$. The main purpose of the present paper is to derive a solution to the optimal stopping problem for the (time-homogeneous strong) Markov process $(X, Y)$ given by:

$$
V_{*}(x, y)=\inf _{\tau} E_{x, y}\left[e^{r \tau}\left(Y_{\tau}+L\right)\right]
$$

where the infimum is taken over finite stopping times $\tau$ with respect to the natural filtration $\left(\mathcal{F}_{t}\right)_{t \geq 0}$ of the process $X$, and $L>0$ is fixed. Here $E_{x, y}$ denotes the expectation under the assumption that the (twodimensional) process $(X, Y)$ defined in (2.1)-(2.3) starts at $(x, y) \in E$, and $E=\left\{(x, y) \in \mathbb{R}^{2} \mid 0<y \leq x\right\}$ is the state space of the process $(X, Y)$. We observe from the structure of the reward in the expression of (2.4) that the infimum there is actually taken over finite stopping times $\tau$ of the process $X$ for which the condition:

$$
E_{x, y}\left[e^{r \tau}\right]<\infty
$$

holds. (Note that, due to the assumption that $L>0$, the condition of $(2.5)$ turns out to be stronger than the finiteness of the expected reward in (2.4) under $L=0$ which is used in [38] and [13]. The verification of this condition is very complicated and is actually skipped below for the cases of different relations between the parameters of the model considered in the paper.) The operations of such contracts can be described as follows. It is assumed that the short sellers receive the fixed payments $V_{*}(x, y)$ at time 0 and incur obligations to deliver to the buyers the payoffs $e^{r \tau}\left(Y_{\tau}+L\right)$ at some future times $\tau$ which the sellers can choose. In other words, the sellers can obtain the assets at the best prices $Y_{\tau}$ they could have obtained up to the time of exercise $\tau$ with some additional fixed fee $L>0$, but paid in the equivalent currency units at time 0 , that is, $e^{r \tau}\left(Y_{\tau}+L\right)$ (see [38] for the precise extensive formulation of the contract under $L=0$ ). We recall that the problem of (2.4) for the case of $L=0$ was actually solved is [38] (see also [13] for a solution by means of the change-of-measure arguments following [37]). Moreover, it follows from the result of [38; Section 2, Lemma] that, if $0<r \leq\left(\mu-\sigma^{2} / 2\right)^{2} /\left(2 \sigma^{2}\right)$ and $\mu<\sigma^{2} / 2$ holds, then $V_{*}(x, y)=0$, for all $(x, y) \in E$, under $L=0$. In this respect, we further consider the cases in which either $\mu>\sigma^{2} / 2$ or $r>\left(\mu-\sigma^{2} / 2\right)^{2} /\left(2 \sigma^{2}\right)$ holds. The optimal stopping problem dual to the one of (2.4) was solved in [28] and [22] for the case of an underlying geometric Brownian motion (see also [11] for the case of an underlying jump-diffusion process).

2.2 Structure of the optimal stopping time. Let us first determine the structure of the optimal stopping time in the problem of (2.4).

(i) By means of standard applications of Itô's formula (see, e.g. [24; Chapter III, Theorem 3.3] and [35; Chapter II, Theorem 3.2]) to the process $e^{r t}\left(Y_{t}+L\right)$, we obtain the representation:

$$
e^{r t}\left(Y_{t}+L\right)=y+L+\int_{0}^{t} e^{r s} r\left(Y_{s}+L\right) I\left(X_{s} \neq Y_{s}\right) d s+\int_{0}^{t} e^{r s} I\left(X_{s}=Y_{s}\right) d Y_{s}
$$

for all $t \geq 0$. Then, inserting $\tau$ instead of $t$ and taking the expectation with respect to the probability measure $P_{x, y}$ in (2.6), we get that the expected reward from (2.4) admits the representation:

$$
E_{x, y}\left[e^{r \tau}\left(Y_{\tau}+L\right)\right]=y+L+E_{x, y}\left[\int_{0}^{\tau} e^{r s} r\left(Y_{s}+L\right) I\left(X_{s} \neq Y_{s}\right) d s+\int_{0}^{\tau} e^{r s} I\left(X_{s}=Y_{s}\right) d Y_{s}\right]
$$

for any stopping time $\tau$ satisfying the condition of $(2.5)$, where $I(\cdot)$ denotes the indicator function.

Let us now apply some heuristic arguments based on the probabilistic properties of the running minima of geometric Brownian motions which are not directly used in the subsequent verification in the proof of Theorem 4.1 below. More precisely, by virtue of properties of the running minimum $Y$ from (2.3) of the geometric Brownian motion $X$ from (2.1)-(2.2) (see, e.g. [10; Subsection 3.3] for similar arguments applied to the running maxima of the Bessel processes), it follows that, for some $y^{\prime}>0$ fixed and an infinitesimally small deterministic time interval $\Delta$, we have:

$$
Y_{\Delta}=y^{\prime} \wedge \min _{0 \leq s \leq \Delta} X_{s}=y^{\prime} \wedge\left(y^{\prime}+\Delta X\right)+o(\Delta) \quad \text { as } \Delta \downarrow 0
$$


where we set $\Delta X=X_{\Delta}-y^{\prime}$. Observe that $\Delta Y=o(\Delta)$ when $\Delta X \geq 0$, and $\Delta Y=\Delta X+o(\Delta)$ when $\Delta X<0$, where we set $\Delta Y=Y_{\Delta}-y^{\prime}$ and recall that $o(\Delta)$ denotes a random function satisfying $o(\Delta) / \Delta \rightarrow 0$ as $\Delta \downarrow 0$ $(P$-a.s. $)$. In this case, using the asymptotic formula:

$$
E_{y^{\prime}, y^{\prime}}[\Delta X ; \Delta X<0] \equiv E_{y^{\prime}, y^{\prime}}[\Delta X I(\Delta X<0)] \sim-y^{\prime} \sqrt{\frac{\Delta}{2 \pi}} \quad \text { as } \Delta \downarrow 0
$$

as well as applying the representation in (2.7), we get:

$$
E_{y^{\prime}, y^{\prime}}\left[e^{r \Delta} r\left(y^{\prime}+L\right) \Delta+e^{r \Delta} \Delta Y\right] \sim e^{r \Delta} r\left(y^{\prime}+L\right) \Delta-e^{r \Delta} y^{\prime} \sqrt{\frac{\Delta}{2 \pi}} \quad \text { as } \Delta \downarrow 0
$$

for each $y^{\prime}>0$ fixed.

It follows from the results of general theory of optimal stopping problems for Markov processes (see, e.g. [33; Chapter I, Subsection 2.2]) that the continuation and stopping regions of the optimal stopping problems in (2.4) should have the form:

$$
C_{*}=\left\{(x, y) \in E \mid V_{*}(x, y)<y+L\right\} \quad \text { and } \quad D_{*}=\left\{(x, y) \in E \mid V_{*}(x, y)=y+L\right\} .
$$

(It is seen from the solution presented in Theorem 4.1 below that $V_{*}(x, y)$ is a continuous function, so that the set $C_{*}$ is open and the set $D_{*}$ is closed.) Then, we observe from the expressions in (2.7) and (2.10) that it is never optimal to stop the process $(X, Y)$ when $X_{t}=Y_{t}$, for each $t \geq 0$, so that all the points of the diagonal $\left\{(x, y) \in \mathbb{R}^{2} \mid 0<x=y\right\}$ belong to the continuation region $C_{*}$ in (2.11). Moreover, it follows from the definition of the process $(X, Y)$ in (2.1)-(2.3) and the structure of the reward in (2.4) that, for each $y>0$ fixed, there exists a sufficiently large $x>0$ such that the point $(x, y)$ belongs to the stopping region $D_{*}$ in (2.11). According to arguments similar to the ones applied in [10; Subsection 3.3] and [29; Subsection 3.3], the latter property can be explained by the fact that the costs of waiting until the process $X$ coming from such a large $x>0$ decreases the current value of the running minimum process $Y$ may be too large due to the presence of the discounting factor in the reward functional of (2.4).

(ii) Let us denote by $\tau_{*}=\tau_{*}(x, y)$ the optimal stopping time in the problem of (2.4) given that the process $(X, Y)$ starts at the point $(x, y) \in E$. Consider some point $(x, y) \in C_{*}$ and take another starting point $\left(x^{\prime}, y\right)$ for the process $(X, Y)$ such that $0<y \leq x^{\prime}<x$. Then, using the fact that the running minimum $Y$ from (2.3) of the process $X$ from (2.1) started at the point $x^{\prime}$ is less or equal to the running minimum started at $x$, we obtain:

$$
\begin{aligned}
& V_{*}\left(x^{\prime}, y\right)-(y+L) \leq E_{x^{\prime}, y}\left[e^{r \tau_{*}}\left(Y_{\tau_{*}}+L\right)\right]-(y+L) \\
& \leq E_{x, y}\left[e^{r \tau_{*}}\left(Y_{\tau_{*}}+L\right)\right]-(y+L)=V_{*}(x, y)-(y+L)<0
\end{aligned}
$$

so that $\left(x^{\prime}, y\right) \in C_{*}$. On the other hand, if we take some point $(x, y) \in D_{*}$ from (2.11) and use the fact that the process $(X, Y)$ started at some point $\left(x^{\prime \prime}, y\right)$ such that $x^{\prime \prime}>x \geq y$ passes through $(x, y)$ before hitting the diagonal $\left\{(x, y) \in \mathbb{R}^{2} \mid 0<x=y\right\}$, then the equalities in (2.4) and (2.7) imply that $V_{*}\left(x^{\prime \prime}, y\right)-(y+L) \geq V_{*}(x, y)-(y+L)=0$ holds, so that $\left(x^{\prime \prime}, y\right) \in D_{*}$. Hence, combining these arguments together with the comments in [10; Subsection 3.3] and [29; Subsection 3.3], we may therefore conclude that there exists a function $h_{*}(y)$, for $y>0$, such that the continuation and stopping regions $C_{*}$ and $D_{*}$ in (2.11) take the form:

$$
C_{*}=\left\{(x, y) \in E \mid x<h_{*}(y)\right\} \quad \text { and } \quad D_{*}=\left\{(x, y) \in E \mid x \geq h_{*}(y)\right\}
$$

respectively. (Note that the existence of such a boundary $h_{*}(y)$ can also be deduced from the concavity and monotonicity of the function $x \mapsto V_{*}(x, y)$ on $(y, \infty)$, for each $y>0$ fixed.)

(iii) Let us now determine the location of the optimal stopping boundary $h_{*}(y)$. For this purpose, we denote by $U_{*}(x, y)$ the value function of the optimal stopping problem which can be obtained from the one in (2.4) above or (5.1) below, by means of setting $L=0$ there. It is shown in [38] (see also [13] for another derivation) that the function $U_{*}(x, y) \equiv V_{*}(x, y ; 0) \equiv W_{*}(x, y ; 0)$ with $V_{*}(x, y) \equiv V_{*}(x, y ; L)$ from $(2.4)$ and $W_{*}(x, y) \equiv W_{*}(x, y ; L)$ from (5.1) admits the explicit expression in (5.16) below, under $L=0$, and the optimal stopping time has the form $\eta_{*}=\inf \left\{t \geq 0 \mid X_{t} \geq a_{*} Y_{t}\right\}$, where $a_{*}$ is defined by $a_{*}=b_{*}(0)$ with $b_{*} \equiv b_{*}(L)$ determined from the expressions in (5.11), or (5.13), or (5.15), respectively (see Theorem 5.1 


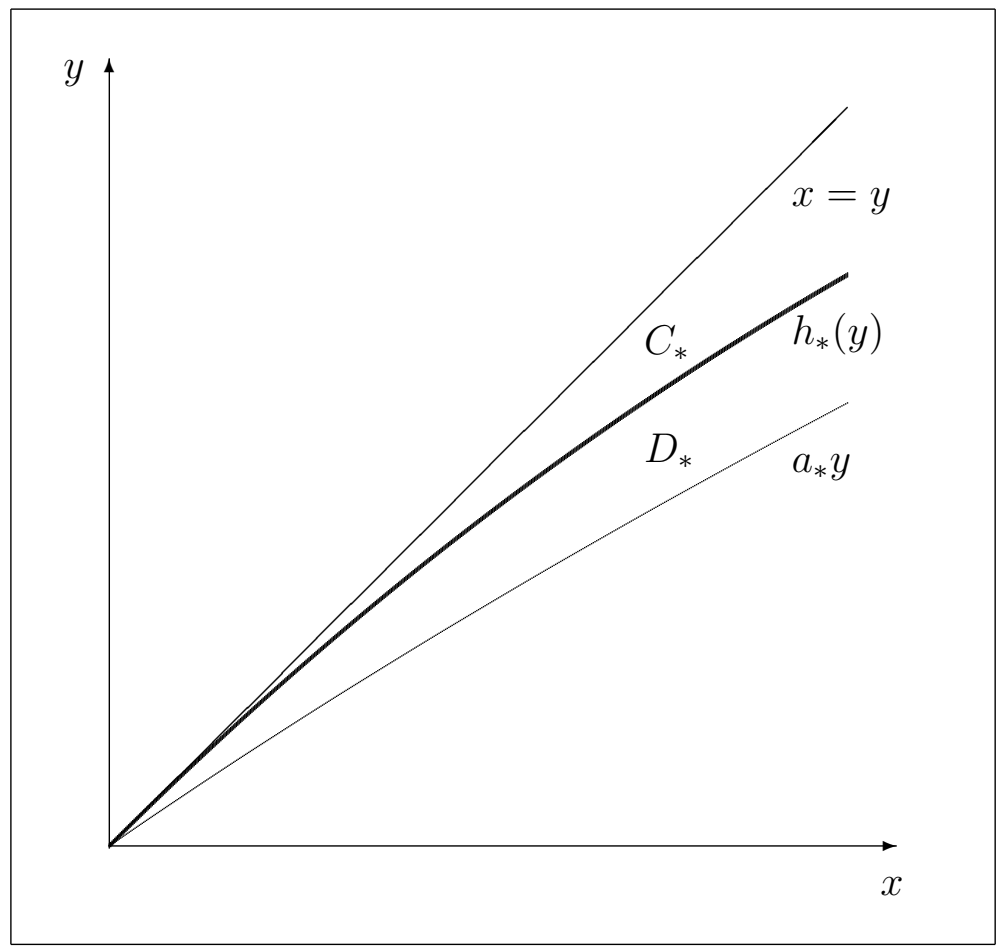

Figure 1. A computer drawing of the optimal stopping boundary $h_{*}(y)$.

below). (Note that $a_{*}$ here corresponds to $\theta$ in the notation of [38] and to $1 / a_{*}$ in the notations of [13].) Suppose that $h_{*}(y)>a_{*} y$ holds, for some $y>0$. Then, for each $x \in\left(a_{*} y, h_{*}(y)\right)$ given and fixed, we would have $V_{*}(x, y)<y+L=U_{*}(x, y)+L$, contradicting the obvious fact that $U_{*}(x, y)+L \leq V_{*}(x, y)$, for all $(x, y) \in E$, as it is clearly seen from the structure of the payoff in (2.4). Thus, we may conclude that $y<h_{*}(y) \leq a_{*} y$ should hold, for all $y>0$ (see Figure 1 above for a computer drawing of the optimal stopping boundary $\left.h_{*}(y)\right)$.

2.3 The free-boundary problem. By means of standard arguments based on the application of Itô's formula (see, e.g. [24; Chapter V, Section 5.1]), it is shown that the infinitesimal operator $\mathbb{L}$ of the process $(X, Y)$ from $(2.1)-(2.3)$ acts on an arbitrary function $F(x, y)$ from the class $C^{2,1}$ on $E$ according to the rule:

$$
\begin{aligned}
& (\mathbb{L} F)(x, y)=\mu x \partial_{x} F(x, y)+\left(\sigma^{2} x^{2} / 2\right) \partial_{x x} F(x, y) \text { in } 0<y<x \\
& \partial_{y} F(x, y)=0 \text { at } x=y
\end{aligned}
$$

(see, e.g. [29; Subsection 3.1]). In order to find analytic expressions for the unknown value functions $V_{*}(x, y)$ from (2.4) and the unknown boundary $h_{*}(y)$ from (2.13), we use the results of general theory of optimal stopping problems for Markov processes (see, e.g. [33; Chapter IV, Section 8] as well as [33; Chapter V, Sections 15-20] and references therein for optimal stopping problems for maximum processes). More precisely, we reduce the optimal stopping problem of (2.4) to the equivalent free-boundary problem:

$$
\begin{aligned}
& (\mathbb{L} V+r V)(x, y)=0 \quad \text { for } \quad(x, y) \in C \quad \text { such that } \quad x \neq y \\
& \left.V(x, y)\right|_{x=h(y)-}=y+L \quad(\text { instantaneous stopping }) \\
& \left.\partial_{x} V(x, y)\right|_{x=h(y)-}=0 \quad(\text { smooth fit }) \\
& \left.\partial_{y} V(x, y)\right|_{x=y+}=0 \quad(\text { normal reflection }) \\
& V(x, y)=y+L \quad \text { for } \quad(x, y) \in D \\
& V(x, y)<y+L \quad \text { for } \quad(x, y) \in C \\
& (\mathbb{L} V+r V)(x, y)>0 \quad \text { for } \quad(x, y) \in D
\end{aligned}
$$

where $C$ and $D$ are defined as $C_{*}$ and $D_{*}$ in $(2.13)$ with $h(y)$ instead of $h_{*}(y)$, respectively. Observe that the subharmonic characterisation of the value function (see, e.g. [33; Chapter IV, Section 9]) implies that $V_{*}(x, y)$ are the largest function satisfying (2.16)-(2.17) and (2.20)-(2.21) with the boundary $h_{*}(y)$. Here, the conditions of (2.17)-(2.19) are satisfied, for all $y>0$. We recall that, in the case of $L=0$, the free-boundary problem (2.16)-(2.22) was actually solved in [38] (see also [13] for another derivation). 


\section{Solution to the free-boundary problem}

In this section, we derive analytic solutions to the free-boundary problem stated in (2.16)-(2.22). For this purpose, we consider three separate cases based on the different relations between the parameters of the model.

3.1 The case $0<r<\left(\mu-\sigma^{2} / 2\right)^{2} /\left(2 \sigma^{2}\right)$ and $\mu>\sigma^{2} / 2$. Let us first assume that $0<r<\left(\mu-\sigma^{2} / 2\right)^{2} /\left(2 \sigma^{2}\right)$ and $\mu>\sigma^{2} / 2$ holds. Then, the general solution of the second-order ordinary differential equation in (2.16) has the form:

$$
V(x, y)=C_{1}(y) x^{\gamma_{1}}+C_{2}(y) x^{\gamma_{2}}
$$

for all $y<x<h(y)$, where $C_{j}(y), j=1,2$, are some arbitrary (continuously differentiable) functions, and $\gamma_{j}, j=1,2$, are given by:

$$
\gamma_{j}=\frac{1}{2}-\frac{\mu}{\sigma^{2}}-(-1)^{j} \sqrt{\left(\frac{1}{2}-\frac{\mu}{\sigma^{2}}\right)^{2}-\frac{2 r}{\sigma^{2}}}
$$

so that $\gamma_{2}<\gamma_{1}<0$ in this case. (Note that $\gamma_{j}, j=1,2$, in (3.2) are the same as in the notations of [38] and correspond to $1-\eta_{j}, j=1,2$, in the notations of [13].) Then, by applying the conditions of (2.17)-(2.19) to the function in (3.1), we obtain that the equalities:

$$
\begin{aligned}
& C_{1}(y) h^{\gamma_{1}}(y)+C_{2}(y) h^{\gamma_{2}}(y)=y+L \\
& C_{1}(y) \gamma_{1} h^{\gamma_{1}}(y)+C_{2}(y) \gamma_{2} h^{\gamma_{2}}(y)=0 \\
& C_{1}^{\prime}(y) y^{\gamma_{1}}+C_{2}^{\prime}(y) y^{\gamma_{2}}=0
\end{aligned}
$$

should hold, for all $y>0$. Hence, by solving the system in (3.3)-(3.5), we obtain that the candidate value function has the form:

$$
V(x, y ; h(y))=(y+L)\left(\frac{\gamma_{2}}{\gamma_{2}-\gamma_{1}}\left(\frac{x}{h(y)}\right)^{\gamma_{1}}-\frac{\gamma_{1}}{\gamma_{2}-\gamma_{1}}\left(\frac{x}{h(y)}\right)^{\gamma_{2}}\right)
$$

for $y \leq x<h(y)$, and the candidate boundary satisfies the ordinary differential equation:

$$
h^{\prime}(y)=\frac{h(y)}{y+L} \frac{\gamma_{2}(y / h(y))^{\gamma_{1}}-\gamma_{1}(y / h(y))^{\gamma_{2}}}{\gamma_{1} \gamma_{2}\left((y / h(y))^{\gamma_{1}}-(y / h(y))^{\left.\gamma_{2}\right)}\right.}
$$

for $y>0$. Moreover, by means of straightforward computations, it can be deduced from the expression in (3.6) that the first- and second-order partial derivatives $\partial_{x} V(x, y ; h(y))$ and $\partial_{x x} V(x, y ; h(y))$ of the function $V(x, y ; h(y))$ take the form:

$$
\partial_{x} V(x, y ; h(y))=\frac{\gamma_{1} \gamma_{2}(y+L)}{\left(\gamma_{2}-\gamma_{1}\right) h(y)}\left(\left(\frac{x}{h(y)}\right)^{\gamma_{1}-1}-\left(\frac{x}{h(y)}\right)^{\gamma_{2}-1}\right)
$$

and

$$
\partial_{x x} V(x, y ; h(y))=\frac{\gamma_{1} \gamma_{2}(y+L)}{\left(\gamma_{2}-\gamma_{1}\right) h^{2}(y)}\left(\left(\gamma_{1}-1\right)\left(\frac{x}{h(y)}\right)^{\gamma_{1}-2}-\left(\gamma_{2}-1\right)\left(\frac{x}{h(y)}\right)^{\gamma_{2}-2}\right)
$$

on the interval $y \leq x<h(y)$, for each $y>0$ fixed.

3.2 The case $r=\left(\mu-\sigma^{2} / 2\right)^{2} /\left(2 \sigma^{2}\right)$ and $\mu>\sigma^{2} / 2$. Let us now assume that $r=\left(\mu-\sigma^{2} / 2\right)^{2} /\left(2 \sigma^{2}\right)$ and $\mu>\sigma^{2} / 2$ holds. Then, the general solution of the ordinary differential equation in (2.16) has the form:

$$
V(x, y)=C_{1}(y) x^{\alpha} \ln x+C_{2}(y) x^{\alpha}
$$

for all $y<x<h(y)$, where $C_{j}(y), j=1,2$, are some arbitrary functions, and $\alpha$ is given by:

$$
\alpha=\frac{1}{2}-\frac{\mu}{\sigma^{2}}
$$


so that $\alpha<0$ in this case. (Note that $\alpha$ in (3.11) is the same as in the notations of [38] and corresponds to $1-\alpha$ on the notations of [13].) Then, by applying the conditions from (2.17)-(2.19) to the function in (3.10), we get that the equalities:

$$
\begin{aligned}
& C_{1}(y) h^{\alpha}(y) \ln h(y)+C_{2}(y) h^{\alpha}(y)=y+L \\
& C_{1}(y) \alpha h^{\alpha}(y) \ln h(y)+C_{1}(y) h^{\alpha}(y)+C_{2}(y) \alpha h^{\alpha}(y)=0 \\
& C_{1}^{\prime}(y) y^{\alpha} \ln y+C_{2}^{\prime}(y) y^{\alpha}=0
\end{aligned}
$$

should hold, for all $y>0$. Hence, by solving the system in (3.12)-(3.14), we obtain that the candidate value function has the form:

$$
V(x, y ; h(y))=(y+L)\left(\frac{x}{h(y)}\right)^{\alpha}\left(1-\alpha \ln \left(\frac{x}{h(y)}\right)\right)
$$

for $y \leq x<h(y)$, and the candidate boundary satisfies the ordinary differential equation:

$$
h^{\prime}(y)=\frac{h(y)}{y+L} \frac{\alpha \ln (y / h(y))-1}{\alpha^{2} \ln (y / h(y))}
$$

for $y>0$.

3.3 The case $r>\left(\mu-\sigma^{2} / 2\right)^{2} /\left(2 \sigma^{2}\right)$. Let us finally assume that $r>\left(\mu-\sigma^{2} / 2\right)^{2} /\left(2 \sigma^{2}\right)$ holds. Then, the general solution of the ordinary differential equation in (2.16) has the form:

$$
V(x, y)=C_{1}(y) x^{\alpha} \sin (\beta \ln x)+C_{2}(y) x^{\alpha} \cos (\beta \ln x)
$$

for all $y<x<h(y)$, where $C_{j}(y), j=1,2$, are some arbitrary functions, while $\alpha$ is given by (3.11) and $\beta$ is defined as:

$$
\beta=\sqrt{\frac{2 r}{\sigma^{2}}-\left(\frac{1}{2}-\frac{\mu}{\sigma^{2}}\right)^{2}} .
$$

(Note that $\beta$ in (3.18) is the same as in the notations of [38] and [13].) Then, by applying the conditions from (2.17)-(2.19) to the function in (3.17), we get that the equalities:

$$
\begin{aligned}
& C_{1}(y) h^{\alpha}(y) \sin (\beta \ln h(y))+C_{2}(y) h^{\alpha}(y) \cos (\beta \ln h(y))=y+L \\
& \left(C_{1}(y) \alpha-C_{2}(y) \beta\right) h^{\alpha}(y) \sin (\beta \ln h(y))+\left(C_{1}(y) \beta+C_{2}(y) \alpha\right) h^{\alpha}(y) \cos (\beta \ln h(y))=0 \\
& C_{1}^{\prime}(y) y^{\alpha} \sin (\beta \ln y)+C_{2}^{\prime}(y) y^{\alpha} \cos (\beta \ln y)=0
\end{aligned}
$$

should hold, for all $y>0$. Hence, by solving the system in (3.19)-(3.21), we obtain that the candidate value function has the form:

$$
V(x, y ; h(y))=(y+L)\left(\frac{x}{h(y)}\right)^{\alpha}\left(\cos \left(\beta \ln \left(\frac{x}{h(y)}\right)\right)-\frac{\alpha}{\beta} \sin \left(\beta \ln \left(\frac{x}{h(y)}\right)\right)\right)
$$

for $y \leq x<h(y)$, and the candidate boundary satisfies the ordinary differential equation:

$$
h^{\prime}(y)=\frac{h(y)}{y+L} \frac{\alpha-\beta \tan (\beta(\ln (y / h(y)))}{\alpha^{2}+\beta^{2}}
$$

for $y>0$.

3.4 The minimal admissible solutions $h_{*}(y)$. We further consider the minimal admissible solutions $h_{*}(y)$ of first-order nonlinear ordinary differential equations as the smallest possible solutions of the equations in (3.7), (3.16), and (3.23) which satisfy the inequality $h_{*}(y)>y$, for all $y>0$. By virtue of the classical results on the existence and uniqueness of solutions for first-order nonlinear ordinary differential equations, we may conclude that these equations admit (locally) unique solutions, in view of the facts that the righthand sides in (3.7), (3.16), and (3.23) are (locally) continuous in $(y, h(y))$ and (locally) Lipschitz in $h(y)$, for each $y>0$ fixed (see, e.g. [29; Subsection 3.9]). Then, it is shown by means of technical arguments based 
on Picard's method of successive approximations that there exists a unique solution $h(y)$ to the equation in (3.7), or (3.16), or (3.23), under the corresponding relations between the parameters of the model, for $y>0$, started at some point $\left(y_{0}, y_{0}\right)$ such that $y_{0}>0$ (see also [21; Subsection 3.2] and [29; Example 4.4] for similar arguments based on the analysis of other first-order nonlinear ordinary differential equations, as well as the solutions of the corresponding ordinary differential equations in [38; Formulae (2.8), (2.10), and (2.11)], under $L=0)$. Hence, in order to construct the appropriate function $h_{*}(y)$ which satisfies the equation in $(3.7)$, or (3.16), or (3.23), and stays strictly above the diagonal, we can follow the arguments from [32; Subsection 3.5] (among others) which are based on the construction of sequences of the so-called bad-good solutions which intersect the diagonal. For this purpose, for any sequence $\left(y_{l}\right)_{l \in \mathbb{N}}$ such that $y_{l}>0$ and $y_{l} \uparrow \infty$ as $l \rightarrow \infty$, we can construct the sequence of solutions $h_{l}(y), l \in \mathbb{N}$, to the equation in (3.7), or (3.16), or (3.23), for all $y>0$ such that $h_{l}\left(y_{l}\right)=y_{l}$ holds, for each $l \in \mathbb{N}$. It follows from the structure of these first-order nonlinear ordinary differential equations that the property $h_{l}^{\prime}\left(y_{l}\right)=-\infty$ holds, for each $l \in \mathbb{N}$ (see also [28; pages 979-982] for the analysis of solutions of the single first-order nonlinear differential equation corresponding to the case of lookback put options). Observe that, by virtue of the uniqueness of solutions mentioned above, we know that each two curves $y \mapsto h_{l}(y)$ and $y \mapsto h_{m}(y)$ cannot intersect, for $l, m \in \mathbb{N}, l \neq m$, and thus, we see that the sequence $\left(h_{l}(y)\right)_{l \in \mathbb{N}}$ is increasing, so that the limit $h_{*}(y)=\lim _{l \rightarrow \infty} h_{l}(y)$ exists, for each $y>0$. We may therefore conclude that $h_{*}(y)$ provides the minimal solution to the equation in (3.7), or (3.16), or (3.23) such that $h_{*}(y)>y$ holds, for all $y>0$. Moreover, since the right-hand sides of the first-order nonlinear ordinary differential equations in (3.7), (3.16), and (3.23) are (locally) Lipschitz in $y$, one can deduce by means of Gronwall's inequality that the functions $h_{l}(y), l \in \mathbb{N}$, are continuous, so that the function $h_{*}(y)$ is continuous too. The corresponding maximal admissible solutions of first-order nonlinear ordinary differential equations and the associated maximality principle for solutions of optimal stopping problems which is equivalent to the superharmonic characterisation of the payoff functions were established in [29] and further developed in [21], [28], [22], [11], [6], [23], [31]-[32], [20], [27], [25], [7], [16]-[18], and [34] among other subsequent papers (see also [33; Chapter I; Chapter V, Section 17] for other references).

\section{Main results and proofs}

In this section, we show that the solution of the free-boundary problem from (2.16)-(2.22) found in the previous section provides the solution of the initial optimal stopping problem of (2.4). Such a verification assertion was proved in [38] for the solution of the associated two-dimensional optimal stopping problem in the case $L=0$ (see also [13] for another proof following the arguments of [37] and [39; Chapter VIII, Subsection 2d]).

Theorem 4.1 Let the processes $X$ and $Y$ be given by the expressions in (2.1)-(2.3). Then, the value function of the perpetual dual lookback optimal stopping problem from (2.4) in which the infimum is taken over all finite stopping times $\tau$ of $X$ satisfying the condition of (2.5) admits the representation:

$$
V_{*}(x, y)= \begin{cases}V\left(x, y ; h_{*}(y)\right), & \text { if } y \leq x<h_{*}(y) \\ y+L, & \text { if } x \geq h_{*}(y)\end{cases}
$$

whenever the optimal stopping time being of the form:

$$
\tau_{*}=\inf \left\{t \geq 0 \mid X_{t} \geq h_{*}\left(Y_{t}\right)\right\}
$$

satisfies the condition of (2.5), where the candidate value function and the boundary are specified as follows:

(i) if $0<r<\left(\mu-\sigma^{2} / 2\right)^{2} /\left(2 \sigma^{2}\right)$ and $\mu>\sigma^{2} / 2$ holds, then $V\left(x, y ; h_{*}(y)\right)$ takes the expression of (3.6), for $y \leq x<h_{*}(y)$, and $h_{*}(y)$ is the minimal solution of the (first-order nonlinear) ordinary differential equation in (3.7) satisfying $y<h_{*}(y)\left[\leq a_{*} y\right]$, for $y>0$, with $a_{*}=b_{*}(0)$ from (5.11);

(ii) if $r=\left(\mu-\sigma^{2} / 2\right)^{2} /\left(2 \sigma^{2}\right)$ and $\mu>\sigma^{2} / 2$ holds, then $V\left(x, y ; h_{*}(y)\right)$ takes the expression of (3.15), for $y \leq x<h_{*}(y)$, and $h_{*}(y)$ is the minimal solution of the ordinary differential equation in (3.16) satisfying $y<h_{*}(y)\left[\leq a_{*} y\right]$, for $y>0$, with $a_{*}=b_{*}(0)$ from (5.13);

(iii) if $r>\left(\mu-\sigma^{2} / 2\right)^{2} /\left(2 \sigma^{2}\right)$ holds, then $V\left(x, y ; h_{*}(y)\right)$ takes the expression of (3.22), for $y \leq x<h_{*}(y)$, and $h_{*}(y)$ is the minimal solution of the ordinary differential equation in (3.23) satisfying $y<h_{*}(y)\left[\leq a_{*} y\right]$, for $y>0$, with $a_{*}=b_{*}(0)$ from (5.15). 
Proof: In order to verify the assertions stated above, let us show that the function defined in (4.1) coincides with the value function in (2.4) and the stopping time $\tau_{*}$ from (4.2) is optimal under the assumption that is satisfies the condition of (2.5). For this purpose, let us consider any solution $h(y)$ of the ordinary differential equation in (3.7), or (3.16), or (3.23) such that $h(y)>y$, for all $y>0$. We thus denote by $V_{h}(x, y)$ the righthand side of the expression in (4.1) associated with $h(y)$. Then, by means of straightforward calculations of the previous section, it is shown that the function $V_{h}(x, y)$ solves the system of (2.16) with (2.20)-(2.22) and satisfies the conditions of (2.17)-(2.19). We also recall that the function $V_{h}(x, y)$ is $C^{2,1}$ in $C$ and $D$ which are defined as $C_{*}$ and $D_{*}$ in $(2.13)$ with $h(y)$ instead of $h_{*}(y)$, respectively. Hence, taking into account the assumption that $h(y)$ is continuously differentiable, by applying the change-of-variable formula from [30] to the process $e^{r t} V_{h}\left(X_{t}, Y_{t}\right)$ (see also [33; Chapter II, Section 3.5] for a summary of the related results on the local time-space formula as well as further references), we obtain:

$$
\begin{aligned}
e^{r t} V_{h}\left(X_{t}, Y_{t}\right)= & V_{h}(x, y)+\int_{0}^{t} e^{r s}\left(\mathbb{L} V_{h}+r V_{h}\right)\left(X_{s}, Y_{s}\right) I\left(X_{s} \neq h\left(Y_{s}\right), X_{s} \neq Y_{s}\right) d s \\
& +\int_{0}^{t} e^{r s} \partial_{y} V_{h}\left(X_{s}, Y_{s}\right) I\left(X_{s}=Y_{s}\right) d Y_{s}+M_{t}
\end{aligned}
$$

for all $t \geq 0$, where the process $M=\left(M_{t}\right)_{t \geq 0}$ defined by:

$$
M_{t}=\int_{0}^{t} e^{r s} \partial_{x} V_{h}\left(X_{s}, Y_{s}\right) I\left(X_{s} \neq Y_{s}\right) \sigma X_{s} d B_{s}
$$

is a continuous local martingale with respect to the probability measure $P_{x, y}$. Note that, since the time spent by the process $(X, Y)$ at the boundary surface $\{(x, y) \in E \mid x=h(y)\}$ as well as at the diagonal $\left\{(x, y) \in \mathbb{R}^{2} \mid 0<x=y\right\}$ is of Lebesgue measure zero, the indicator in the first line of the formula in (4.3) as well as in the expression of (4.4) can be ignored. Moreover, since the component $Y$ decreases only when the process $(X, Y)$ is located on the diagonal $\left\{(x, y) \in \mathbb{R}^{2} \mid 0<x=y\right\}$, the indicator in the second line of (4.3) can be set equal to one. Finally, taking into account the fact that the function $V_{h}(x, y)$ satisfies the normal reflection condition of (2.19) at the diagonal, we may conclude that the integral in the second line of (4.3) is actually equal to zero.

It follows from straightforward calculations and the arguments from the previous section that the function $V_{h}(x, y)$ satisfies the second-order ordinary differential equation in (2.16), which together with the conditions of (2.17)-(2.18) and (2.20) as well as the obvious fact that the inequality in (2.22) holds imply that the inequality $\left(\mathbb{L} V_{h}+r V_{h}\right)(x, y) \geq 0$ holds, for any $(x, y) \in E$ such that $x \neq h(y)$ and $x \neq y$, as well. Furthermore, we observe directly from the expressions in (3.6) with (3.8)-(3.9), and it can be shown by means of the same methodology from the expressions in (3.15) and (3.22), that the function $V_{h}(x, y)$ is concave and increases to $y+L$, because its first-order partial derivative $\partial_{x} V_{h}(x, y)$ is positive and decreases to zero, while its second-order partial derivative $\partial_{x x} V_{h}(x, y)$ is negative, on the interval $y \leq x<h(y)$, for each $y>0$ fixed, under the corresponding relations between the parameters of the model considered in the previous section (see also [13; Subsection 4.2] for similar computations related to the case $L=0$ ). Thus, we may conclude that the inequality in (2.21) holds, which together with the conditions of (2.17)-(2.18) and (2.20) imply that the inequality $V_{h}(x, y) \leq y+L$ holds, for all $(x, y) \in E$, too (see also [15; Section 3] and [12; Section 3] for corresponding arguments applied for solutions of other optimal stopping problems). Let $\left(\varkappa_{n}\right)_{n \in \mathbb{N}}$ be the localising sequence of stopping times for the process $M$ from (4.4) such that $\varkappa_{n}=\inf \left\{t \geq 0|| M_{t} \mid \geq n\right\}$, for each $n \in \mathbb{N}$. It therefore follows from the expression in (4.3) that the inequalities:

$$
e^{r\left(\tau \wedge \varkappa_{n}\right)}\left(Y_{\tau \wedge \varkappa_{n}}+L\right) \geq e^{r\left(\tau \wedge \varkappa_{n}\right)} V_{h}\left(X_{\tau \wedge \varkappa_{n}}, Y_{\tau \wedge \varkappa_{n}}\right) \geq V_{h}(x, y)+M_{\tau \wedge \varkappa_{n}}
$$

hold, for any finite stopping time $\tau$ of the process $X$ and each $n \in \mathbb{N}$. Then, taking the expectation with respect to $P_{x, y}$ in (4.5), by means of Doob's optional sampling theorem (see, e.g. [24; Chapter I, Theorem 3.22] and [35; Chapter II, Theorem 3.2]), we get that the inequalities:

$$
E_{x, y}\left[e^{r\left(\tau \wedge \varkappa_{n}\right)}\left(Y_{\tau \wedge \varkappa_{n}}+L\right)\right] \geq E_{x, y}\left[e^{r\left(\tau \wedge \varkappa_{n}\right)} V_{h}\left(X_{\tau \wedge \varkappa_{n}}, Y_{\tau \wedge \varkappa_{n}}\right)\right] \geq V_{h}(x, y)+E_{x, y}\left[M_{\tau \wedge \varkappa_{n}}\right]=V_{h}(x, y)
$$

hold, for any stopping time $\tau$ such that the condition of (2.5) is satisfied, and each $n \in \mathbb{N}$, where we recall that $L>0$ is fixed. Hence, taking into account the fact that the process $Y$ defined in (2.3) and started at 
some $y>0$ is decreasing, letting $n$ go to infinity and applying the Lebesgue dominated convergence theorem under the condition of (2.5), we obtain that the inequalities:

$$
E_{x, y}\left[e^{r \tau}\left(Y_{\tau}+L\right)\right] \geq E_{x, y}\left[e^{r \tau} V_{h}\left(X_{\tau}, Y_{\tau}\right)\right] \geq V_{h}(x, y)
$$

are satisfied, for any stopping time $\tau$ satisfying (2.5), and all $(x, y) \in E$. Thus, taking first the infimum over all stopping times $\tau$ such that the condition of (2.5) holds, and then, the supremum over all boundaries $h$ in the expressions of (4.7), we conclude that the inequalities:

$$
\inf _{\tau} E_{x, y}\left[e^{r \tau}\left(Y_{\tau}+L\right)\right] \geq \sup _{h} V_{h}(x, y)=V_{h_{*}}(x, y)
$$

are satisfied, for all $(x, y) \in E$, where $h_{*}(y)$ is the minimal solution of the ordinary differential equation in (3.7), or (3.16), or (3.23) such that $h_{*}(y)>y$, for each $y>0$ fixed. By applying the standard comparison arguments for solutions of ordinary differential equations or verifying directly (see also similar arguments in [33; Chapter VI, Subsection 21.1.3, Remark 23.2] and [12; Remark 4.3] applied for solutions of other optimal stopping problems), we observe that the function $V_{h}(x, y)$ is (strictly) decreasing in the value $h(y)$, for each $y>0$ fixed, we see that the supremum in (4.8) is attained over any sequence of solutions $\left(h_{k}(y)\right)_{k \in \mathbb{N}}$ to the equation in (3.7), or (3.16), or (3.23) such that $h_{k}(y) \downarrow h_{*}(y)$ as $k \rightarrow \infty$, for each $y>0$ fixed. It follows from the (local) uniqueness of the solutions to the ordinary differential equations in (3.7), (3.16), and (3.23) that no distinct solutions intersect, so that the sequence $\left(h_{k}(y)\right)_{k \in \mathbb{N}}$ is decreasing and the limit $h_{*}(y)=\lim _{k \rightarrow \infty} h_{k}(y)$ exists, for each $y>0$ fixed. Since the inequalities in (4.7) hold for $h_{*}(y)$ too, we see that the expression in (4.8) holds, for $h_{*}(y)$ and $(x, y) \in E$ as well. We also note that $V_{h}(x, y)$ in (4.6) is subharmonic for the Markov process $(X, Y)$ on $E$. Thus, taking into account the facts that $V_{h}(x, y)$ is decreasing in $h(y)>y$ and the inequality $V_{h}(x, y) \leq y+L$ holds, for all $(x, y) \in E$, we observe that the selection of the minimal solution $h_{*}(y)$ such that $h_{*}(y)>y$, for all $y>0$, is equivalent to the implementation of the subharmonic characterisation of the value function (largest subharmonic function subdominating the payoff function, cf. [29] or [33; Chapter I; Chapter V, Section 17]).

In order to prove the fact that the boundary $h_{*}(y)$ is optimal, we consider the sequence of stopping times $\tau_{k}$ defined as in (4.2) with $h_{k}(y)$ instead of $h_{*}(y)$, where $h_{k}(y)$ is a solution to the ordinary differential equation in (3.7), or (3.16), or (3.23) such that $h_{k}(y)>y$ holds, for all $y>0$ and each $k \in \mathbb{N}$. By virtue of the fact that the functions $V_{h_{k}}(x, y)$ from the right-hand side of the expressions in (4.1) associated with the boundaries $h_{k}(y), k \in \mathbb{N}$, satisfy the equation in (2.16) and the condition of (2.17), and taking into account the structure of $\tau_{*}$ in (4.2), it follows from the expression equivalent to the one in (4.3) that the equalities:

$$
e^{r\left(\tau_{k} \wedge \varkappa_{n}\right)}\left(Y_{\tau_{k} \wedge \varkappa_{n}}+L\right)=e^{r\left(\tau_{k} \wedge \varkappa_{n}\right)} V_{h_{k}}\left(X_{\tau_{k} \wedge \varkappa_{n}}, Y_{\tau_{k} \wedge \varkappa_{n}}\right)=V_{h_{k}}(x, y)+M_{\tau_{k} \wedge \varkappa_{n}}
$$

hold, for all $(x, y) \in E$ and each $n, k \in \mathbb{N}$. Hence, taking into account the assumption that the stopping time $\tau_{*}$ from (4.2) satisfies the condition of (2.5) and letting $k$ and $n$ go to infinity as well as using the condition of $(2.17)$ and the property $\tau_{k} \downarrow \tau_{*}\left(P_{x, y}\right.$-a.s. $)$ as $k \rightarrow \infty$, we can apply the Lebesgue dominated convergence theorem to the appropriate (diagonal) subsequence in the expression of (4.9) to obtain the equality:

$$
E_{x, y}\left[e^{r \tau_{*}}\left(Y_{\tau_{*}}+L\right)\right]=E_{x, y}\left[e^{r \tau_{*}} V_{h_{*}}\left(X_{\tau_{*}}, Y_{\tau_{*}}\right)\right]=V_{h_{*}}(x, y)
$$

for all $(x, y) \in E$, which together with the expression in (4.8) directly implies the desired assertion. We finally recall from the results of part (iii) of Subsection 2.2 above implied by standard comparison arguments applied to the value functions of the appropriate optimal stopping problems that the inequality $h_{*}(y) \leq a_{*} y$ should hold for the optimal stopping boundary, that completes the verification.

\section{The case of dual lookback options with floating strikes}

In this section, we present explicit solutions to the optimal stopping problem which is related to the pricing of perpetual dual floating-strike lookback option. 
5.1 The optimal stopping and free-boundary problem. Let us now consider the following optimal stopping problem:

$$
W_{*}(x, y)=\inf _{\zeta} E_{x, y}\left[e^{r \zeta}\left(Y_{\zeta}+L X_{\zeta}\right)\right]
$$

where the infimum is taken over finite stopping times $\zeta$ of the process $X$. Note that the problem of (5.1) was solved in [38] for $L=0$ (see also [13] for a solution by means of the change-of-measure arguments). It can be shown by means of the same arguments as in Subsection 2.2 above that the optimal stopping time in the problem of (5.1) has the structure:

$$
\zeta_{*}=\inf \left\{t \geq 0 \mid X_{t} \geq b_{*} Y_{t}\right\}
$$

with some number $b_{*} \equiv b_{*}(L)>1$. In order to find analytic expressions for the unknown value functions $W_{*}(x, y)$ from $(5.1)$ and the unknown boundaries $b_{*} y$ from (5.2), we can formulate the following free-boundary problem:

$$
\begin{aligned}
& (\mathbb{L} W+r W)(x, y)=0 \quad \text { for } \quad y<x<b y \\
& \left.W(x, y)\right|_{x=(b y)-}=y+\text { Lby } \quad(\text { instantaneous stopping }) \\
& \left.\partial_{x} W(x, y)\right|_{x=(b y)-}=L \quad(\text { smooth fit }) \\
& \left.\partial_{y} W(x, y)\right|_{x=y+}=0 \quad(\text { normal reflection }) \\
& W(x, y)=y+L x \quad \text { for } \quad 0<b y<x \\
& W(x, y)<y+L x \quad \text { for } \quad y \leq x<b y \\
& (\mathbb{L} W+r W)(x, y)>0 \quad \text { for } \quad 0<b y<x
\end{aligned}
$$

where the conditions of (2.17)-(2.19) are satisfied, for all $y>0$. We recall that in the case $L=0$ the free-boundary problem of (5.3)-(5.9) was actually solved in [38] (see also [13] for another derivation).

5.2 Solution to the free-boundary problem. Let us now present the solution of the free-boundary problem in (5.3)-(5.9).

(i) Let us first assume that $0<r<\left(\mu-\sigma^{2} / 2\right)^{2} /\left(2 \sigma^{2}\right)$ and $\mu>\sigma^{2} / 2$ holds. In this case, by using straightforward calculations from Subsection 3.1, it can be shown that the solution of the system in (5.3)(5.9) takes the form:

$$
W\left(x, y ; b_{*} y\right)=\frac{\left(y+L b_{*} y\right) \gamma_{2}-L b_{*} y}{\gamma_{2}-\gamma_{1}}\left(\frac{x}{b_{*} y}\right)^{\gamma_{1}}-\frac{\left(y+L b_{*} y\right) \gamma_{1}-L b_{*} y}{\gamma_{2}-\gamma_{1}}\left(\frac{x}{b_{*} y}\right)^{\gamma_{2}}
$$

for $0<y \leq x<b_{*} y$, and the value $b_{*}$ is determined by the arithmetic equation:

$$
b^{\gamma_{1}-\gamma_{2}}=\frac{\left(\gamma_{1}-1\right)\left(L\left(\gamma_{2}-1\right) b+\gamma_{2}\right)}{\left(\gamma_{2}-1\right)\left(L\left(\gamma_{1}-1\right) b+\gamma_{1}\right)}
$$

(ii) Let us now assume that $r=\left(\mu-\sigma^{2} / 2\right)^{2} /\left(2 \sigma^{2}\right)$ and $\mu>\sigma^{2} / 2$ holds. In this case, by using straightforward calculations from Subsection 3.2, it can be shown that the solution of the system in (5.3)-(5.9) takes the form:

$$
W\left(x, y ; b_{*} y\right)=\left(y+L b_{*} y\right)\left(\left(\frac{x}{b_{*} y}\right)^{\alpha}-\alpha\left(\frac{x}{b_{*} y}\right)^{\alpha} \ln \left(\frac{x}{b_{*} y}\right)^{\alpha}\right)
$$

for $0<y \leq x<b_{*} y$, and the value $b_{*}$ is given by:

$$
b_{*}=\exp \left(\frac{1}{\alpha(\alpha-1)}\right) .
$$

(Note that the latter does not depend on $L$ and coincides with the corresponding optimal boundary in [38] and [13] in this case.) 
(iii) Let us finally assume that $r>\left(\mu-\sigma^{2} / 2\right)^{2} /\left(2 \sigma^{2}\right)$ holds. In this case, by using straightforward calculations from Subsection 3.3, it can be shown that the solution of the system in (5.3)-(5.9) takes the form:

$$
W\left(x, y ; b_{*} y\right)=\left(\frac{x}{b_{*} y}\right)^{\alpha}\left(\left(y+L b_{*} y\right) \cos \left(\beta \ln \left(\frac{x}{b_{*} y}\right)\right)-\frac{\alpha y+(\alpha-1) L b_{*} y}{\beta} \sin \left(\beta \ln \left(\frac{x}{b_{*} y}\right)\right)\right)
$$

for $0<y \leq x<b_{*} y$, and the value $b_{*}$ is determined by the arithmetic equation:

$$
-\cot (\beta \ln b)=\beta(1+L b)+(1-\alpha)(\alpha+L(\alpha-1) b)
$$

Summarising the facts proved above, we formulate the following result which can be proved by means of the same arguments as Theorem 4.1 above (see also proofs of [38; Theorem] and [13; Theorem 4.1]).

Theorem 5.1 Suppose that the assumptions of Theorem 4.1 hold. Then, the value function of the optimal stopping problem in (5.1) admits the representations:

$$
W_{*}(x, y)= \begin{cases}W\left(x, y ; b_{*} y\right), & \text { if } y \leq x<b_{*} y \\ y+L x, & \text { if } x \geq b_{*} y\end{cases}
$$

and the optimal stopping time has the form of (5.2), where the candidate value functions and linear boundaries are specified as follows:

(i): if $0<r<\left(\mu-\sigma^{2} / 2\right)^{2} /\left(2 \sigma^{2}\right)$ and $\mu>\sigma^{2} / 2$ holds, then $W\left(x, y ; b_{*} y\right)$ is given by (5.10), for $y \leq x<$ $b_{*} y$, and while $b_{*}$ is determined by the arithmetic equation in (5.11);

(ii): if $r=\left(\mu-\sigma^{2} / 2\right)^{2} /\left(2 \sigma^{2}\right)$ and $\mu>\sigma^{2} / 2$ holds, then $W\left(x, y ; b_{*} y\right)$ is given by (5.12), for $y \leq x<b_{*} y$, and $b_{*}$ is given by the explicit expression in (5.13);

(iii): if $r>\left(\mu-\sigma^{2} / 2\right)^{2} /\left(2 \sigma^{2}\right)$ holds, then $W\left(x, y ; b_{*} y\right)$ is given by (5.14), for $y \leq x<b_{*} y$, and $b_{*}$ is determined by the arithmetic equation in (5.15).

Acknowledgments. The author is grateful to the Editor and both anonymous Referees for their careful proofreading of the manuscript and valuable suggestions which helped to essentially improve the presentation of the paper.

\section{References}

[1] Asmussen, S., Avram, F. and Pistorius, M. (2003). Russian and American put options under exponential phase-type Lévy models. Stochastic Processes and Applications 109 (79-111).

[2] Avram, F., Kyprianou, A. E. and Pistorius, M. (2004). Exit problems for spectrally negative Lévy processes and applications to (Canadized) Russian options. Annals of Applied Probability 14(1) (215-238).

[3] Battauz, A., De Donno, M. and Sbuelz, A. (2012). Real options with a double continuation region. Quantitative Finance 12(3) (465-475).

[4] Battauz, A., De Donno, M. and Sbuelz, A. (2014). Real options and American derivatives: the double continuation region. Management Science 61(5) (1094-1107).

[5] Beibel, M. and Lerche, H. R. (1997). A new look at warrant pricing and related optimal stopping problems. Empirical Bayes, sequential analysis and related topics in statistics and probability (New Brunswick, NJ, 1995). Statististica Sinica 7 (93-108).

[6] Baurdoux, E. J. and Kyprianou, A. E. (2009). The Shepp-Shiryaev stochastic game driven by a spectrally negative Lévy process. Theory of Probability and its Applications 53 (481-499).

[7] Baurdoux, E. J. and van Schaik, K. (2014). Predicting the time at which a Lévy process attains its ultimate supremum. Acta Applicandae Mathematicae 134(1) (21-44).

[8] De Donno, M., Palmowski, Z. and Tumilewicz, J. (2018). Double continuation regions for American and swing options with negative discount rate in Lévy models. Preprint (24 pp).

[9] Detemple, J. (2006). American-Style Derivatives: Valuation and Computation. Chapman and Hall/CRC, Boca Raton.

[10] Dubins, L., Shepp, L. A. and Shiryaev, A. N. (1993). Optimal stopping rules and maximal inequalities for Bessel processes. Theory of Probability and Applications 38(2) (226-261).

[11] Gapeev, P. V. (2007). Discounted optimal stopping for maxima of some jump-diffusion processes. Journal of Applied Probability 44 (713-731). 
[12] Gapeev, P. V. (2008). The integral option in a model with jumps. Statistics and Probability Letters 76(16) (2623-2631).

[13] Gapeev, P. V. (2019). Solving the dual Russian option problem by using change-of-measure arguments. High Frequency (Special issue in memory of Larry Shepp) 2(2) (76-84).

[14] Gapeev, P. V. (2019). Perpetual dual American barrier options for short sellers. In Stochastic Models, Statistics and Their Applications. SMSA 2019. Steland A., Rafajłowicz E. and Okhrin O. Springer Proceedings in Mathematics and Statistics 294 (85-99).

[15] Gapeev, P. V. and Reiss, M. (2006). An optimal stopping problem in a diffusion-type model with delay. Statistics and Probability Letters 76(6) (601-608).

[16] Gapeev, P. V. and Rodosthenous, N. (2014). Optimal stopping problems in diffusion-type models with running maxima and drawdowns. Journal of Applied Probability 51(3) (799-817).

[17] Gapeev, P. V. and Rodosthenous, N. (2015). On the drawdowns and drawups in diffusion-type models with running maxima and minima. Journal of Mathematical Analysis and Applications 434(1) (413-431).

[18] Gapeev, P. V. and Rodosthenous, N. (2016). Perpetual American options in diffusion-type models with running maxima and drawdowns. Stochastic Processes and their Applications 126(7) (2038-2061).

[19] Gerber, H. U., Michaud, F. and Shiu, E. S. W. (1995). Pricing Russian options with the compound Poisson process. Insurance: Mathematics and Economics 17(1) (79-79).

[20] Glover, K., Hulley, H. and Peskir, G. (2013). Three-dimensional Brownian motion and the golden ratio rule. Annals of Applied Probability 23 (895-922).

[21] Graversen, S. E. and Peskir, G. (1998). Optimal stopping and maximal inequalities for geometric Brownian motion. Journal of Applied Probability 35(4) (856-872).

[22] Guo, X. and Shepp, L. A. (2001). Some optimal stopping problems with nontrivial boundaries for pricing exotic options. Journal of Applied Probability 38(3) (647-658).

[23] Guo, X. and Zervos, M. (2010). $\pi$ options. Stochastic Processes and Applications 120(7) (1033-1059).

[24] Karatzas, I. and Shreve, S. E. (1991). Brownian Motion and Stochastic Calculus. (Second Edition) Springer, New York.

[25] Kyprianou, A. E. and Otт, C. (2014). A capped optimal stopping problem for the maximum process. Acta Applicandae Mathematicae 129 (147-174).

[26] Mordecki, E. and Moreira, W. (2001). Russian options for a difussion with negative jumps. Publicaciones Matemáticas del Uruguay 9 (37-51).

[27] Отт, C. (2013). Optimal stopping problems for the maximum process with upper and lower caps. Annals of Applied Probability 23 (2327-2356).

[28] Pedersen, J. L. (2000). Discounted optimal stopping problems for the maximum process. Journal of Applied Probability 37(4) (972-983).

[29] Peskir, G. (1998). Optimal stopping of the maximum process: The maximality principle. Annals of Probability 26(4) (1614-1640).

[30] Peskir, G. (2007). A change-of-variable formula with local time on surfaces. Séminaire de Probabilité XL. Lecture Notes in Mathematics 1899, Springer (69-96).

[31] Peskir, G. (2012). Optimal detection of a hidden target: The median rule. Stochastic Processes and their Applications $122(2249-2263)$.

[32] Peskir, G. (2014). Quickest detection of a hidden target and extremal surfaces. Annals of Applied Probability 24(6) (2340-2370).

[33] Peskir, G. and Shiryaev, A. N. (2006). Optimal Stopping and Free-Boundary Problems. Birkhäuser, Basel.

[34] Rodosthenous, N. and Zervos, M. (2017). Watermark options. Finance and Stochastics 21(1) (157-186).

[35] Revuz, D. and Yor, M. (1999). Continuous Martingales and Brownian Motion. Springer, Berlin.

[36] Shepp, L. A. and Shinyaev, A. N. (1993). The Russian option: reduced regret. Annals of Applied Probability 3(3) (631-640).

[37] Shepp, L. A. and Shiryaev, A. N. (1994). A new look at the pricing of Russian options. Theory Probability and Applications 39(1) (103-119).

[38] Shepp, L. A. and Shiryaev, A. N. (1996). A dual Russian option for selling short. In Probability Theory and Mathematical Statistics: Lectures presented at the semester held in St. Peterburg, Russia, March 2-April 23, 1993. Ibragimov, I. A. et al. eds. Gordon and Breach, Amsterdam (209-218).

[39] Shiryaev, A. N. (1999). Essentials of Stochastic Finance. World Scientific, Singapore.

[40] XiA, J. and Zhou, X. (2007). Stock loans. Mathematical Finance 17 (307-317). 